\title{
El Paraguay y la acreditación de CARRERAS DE GRADO EN EL MERCOSUR
}

\author{
Higher education acreditation processes in Mercosur \\ and the emergence of nacional evaluation in Paraguay
}

O Paraguai e a ACReditação de Cursos de GraduaÇão NO MERCOSUL

\author{
Gladys Beatriz Barreyro \\ Doutora em Educação. Professora no Programa de Pós-Graduação em Edu- \\ cação da Universidade de São Paulo. São Paulo - SP - Brasil \\ gladysb@usp.br \\ Gabriella de Camargo Hizume \\ Mestre em Integração da América Latina. Professora do curso de Direito da Univer- \\ sidade Estadual do Oeste do Paraná, campus Francisco Beltrão - PR - Brasil \\ ghizume@yahoo.com.br
}

\begin{abstract}
Resumen: El texto analiza los procesos que llevaron a la implementación de la evaluación de la educación superior en Paraguay, mostrando como una política global de evaluación es creada y recreada en las escalas regional y nacional. Se evidencia que fue la adhesión a los procesos de acreditación regional generados en el Mercosur educativo (Mexa y ArcuSur) lo que incentivó y promovió la preocupación en el país por la cuestión y llevó a la creación de una agencia de evaluación nacional, en un contexto de ampliación de instituciones de educación superior, especialmente privadas. El texto es resultado de una investigación bibliográfica, documental y de campo, que contó con entrevistas a participantes de la elaboración y desarrollo de los procesos de evaluación y acreditación.

Palabras Clave: Acreditación de la Educación Superior en Paraguay. Calidad de la Educación Superior. Evaluación de la Educación Superior. Mercosur Educativo. Sistema Arcu-Sur.
\end{abstract}

AвSTRACT: The paper analizes the process of construction of the evaluation of higher education in Paraguay, presenting how a global policy is created and re-created at the regional and national levels. We showed that it was the participation in the regional accreditation processes of the Mercosur educativo (Mexa and Arcu-Sur) which encouraged and promoted the country's concern for evaluation and resulted in the creation of a national evaluation agency, during the expansion of higher education institutions, especially private ones. The paper is based in research, using documental analysis and interviews with participants in the elaboration and development of the evaluation processes.

KeYwords: Higher education evaluation. Accreditation of Higher Education in Paraguay. Mercosur educativo. Quality of higher education. Arcu-Sur System. 
Resumo: O texto analisa o processo de construção da avaliação e da acreditação da educação superior no Paraguai como uma política global, mostrando como tal política é criada e re-criada nas escalas regional e nacional. Evidencia-se que foi a adesão aos processos de acreditação regional gerados no Mercosul educativo (Mexa e Arcu-Sur) o que incentivou e promoveu a preocupação do país pelo assunto e derivou na criação de uma agência de avaliação e acreditação nacional, num marco de expansão de instituiçóes de educação superior, especialmente das privadas. $\mathrm{O}$ texto é baseado em pesquisa de tipo bibliográfica, documental e de campo e inclui dados obtidos de entrevistas mantidas com participantes da elaboração e desenvolvimento dos processos de avaliação.

Palavras-Chave: Acreditação da Educação Superior no Paraguai. Qualidade da Educação Superior. Avaliação da Educação Superior. Mercosul Educacional. Sistema Arcu-Sul.

\section{Introducción}

Desde el inicio del Mercado Común del Sur (Mercosur) existía la preocupación de integrar la educación de los Estados del bloque en todos los niveles. En 1992, en el ańo siguiente a la firma del Tratado de Asunción, que creó el bloque regional, fue instituido el Sector Educativo del Mercosur (SEM), por iniciativa de los Ministros de Educación de los Estados-miembros, para tratar específicamente de las cuestiones del área.

Respecto de la educación superior, el tema de la circulación de trabajadores titulados se presentó como uno de los ejes para profundizar la integración regional. Para hacerlo posible, primeramente era necesario establecer criterios de comparabilidad entre las carreras universitarias de los Estados-miembros y garantizar que cumplieran con los estándares de calidad. Así, empezaron los trabajos para la formulación de un proceso regional de acreditación, entendida cómo certificación de calidad de las carreras universitarias en el Mercosur.

Entre los principales desafíos de esta tarea estaban la elaboración de normas convergentes compatibles con los sistemas nacionales de evaluación y acreditación y la estructuración de órganos para gestionar y ejecutar los procedimientos del proceso regional, en el bloque y nacionalmente. Algunos países del Mercosur como Argentina y Brasil poseían amplia experiencia en el tema, con sistemas nacionales de evaluación de la educación superior consolidados, a pesar de no existir en Brasil la acreditación de carreras de grado; por otro lado, sea por cuestiones históricas o políticas, 
Paraguay no adoptaba la evaluación de grado y Uruguay lo hacía solamente para las instituciones privadas, ya que hasta 2013 había solamente una universidad pública en el país: la Universidad de la República (Udelar).

Para establecer un sistema regional de acreditación en el bloque, primeramente fue elaborado un mecanismo experimental entre los años 2002 y 2006 destinado a las carreras de agronomía, ingeniería y medicina. Este mecanismo fue aplicado en cada país, por órganos indicados por cada uno de los gobiernos, denominados Agencias Nacionales de Acreditación. El proceso de acreditación consistía de tres fases: un procedimiento de autoevaluación, la evaluación externa, realizada por medio de revisión de pares, y el dictamen de acreditación. La gestión del proceso quedó a cargo de la red formada por las Agencias Nacionales de Acreditación, denominada Rana. Con base en esta experiencia, fue creado el Sistema de Acreditación Regional de Carreras Universitarias del Mercosur en 2008. Actualmente, el Sistema Arcu-Sur se encuentra en su segundo ciclo y abarca las carreras de agronomía, arquitectura, economía, enfermería, farmacia, geología, ingeniería, medicina, odontología, veterinaria.

En este escenario, este artículol busca presentar reflexiones sobre la trayectoria de Paraguay en la construcción de su sistema nacional de certificación de calidad de la educación superior a partir de su participación en el proceso regional de acreditación del Mercosur.

\section{Especificidades de la Educación Superior paraguaya}

La educación superior en Paraguay2 incluye grados universitarios y no universitarios, ofrecidos por universidades, institutos superiores y otras instituciones profesionales del tercer nivel, como institutos de formación docente e institutos técnicos (PARAGUAY, 2014a). El nivel superior se caracteriza por su autonomía, a cargo del rectorado de cada institución, independientemente de ser pública o privada. Tanto los cursos de grado como de postgrado (especialización, maestrías y doctorado) se imparten en facultades, institutos o escuelas (PARAGUAY, 2014a). Hasta la década de 1990, Paraguay poseía solamente dos universidades situadas en la capital del país: la Universidad Nacional de Asunción (UNA), fundada en 1889, y la Universidad Católica Nuestra Señora de Asunción, creada en 1960. Por 
el prestigio de las dos únicas instituciones de nivel superior, el control de la calidad del servicio de dichas instituciones era considerado innecesario, puesto que ambas disfrutaban, y aún hoy disfrutan, de inmensa confianza por parte de la sociedad (PEÑA, 2004).

La ampliación del sistema de educación superior comenzó al final de la dictadura militar stronista en el país3, en el año 1989, cuando, según Rivarola (2008), se pasó a cuestionar el rol de la universidad en la formación de la ciudadanía en un régimen democrático y fueron creándose nuevas universidades en diversas localidades del interior, ampliándose la cobertura geográfica de la educación superior y el número de matrículas, propiciando que importantes centros urbanos ofreciesen diversas carreras. Señala Peña (2004) que este período de transición democrática fue marcado por la creación de muchas universidades en un corto período, surgiendo las tres primeras universidades de gestión privada en 1991 y otras tres en el año siguiente.

La apertura de la educación para el sector privado fue consagrada en la Constitución Nacional de 1992. El artículo 74 trata "del derecho de aprender y de la libertad de enseñar", garantizando "la igualdad de oportunidad al acceso a los beneficios de la cultura humanística, de la ciencia y de la tecnología, sin discriminación alguna" y "la libertad de enseñar, sin más requisitos que la idoneidad y la integridad ética, así como el derecho a la educación religiosa y al pluralismo ideológico." Ya el artículo 76 reconoce al sector privado como parte del sistema educativo, aunque la responsabilidad por su organización sea del Estado. Además, se distingue entre el nivel de educación escolar básico, obligatorio y de carácter gratuito en las escuelas oficiales de los otros niveles, entre ellos, el superior y el universitario, que deberán ser fomentados por parte del Estado.

La Constitución también avala una característica muy fuerte de las universidades paraguayas en el artículo 79: la autonomía, garantizada desde la primera Ley de Universidades. La autonomía les confiere la no intervención externa en asuntos universitarios, la amplia libertad para crear nuevas carreras, establecer sus estatutos y su forma de gobierno. En el caso paraguayo, según Rivarola (2008), la autonomía presenta otra faceta: la herencia de concentración de poder y de desreglamentación, lo que resultó para el autor en un proceso de ampliación de la educación superior sin control de la calidad de la enseńanza. 
El sistema universitario paraguayo es considerado de tamaño medio/pequeño出, conteniendo entre 150 a 500 mil matrículas y presentando un nivel de masificación medio-bajo, entre el 26 y el 35\%, en 2008 (BRUNER, HURTADO, 2011)5.

Un dato interesante se refiere al porcentaje de matrículas, pues, a pesar de la proliferación de instituciones privadas, las matrículas se concentraron en el sector estatal (público) inicialmente. En el período 1994 a 2003, la variación de las matrículas fue del 58,8 al 59,9\% en las instituciones públicas y del 41,4 al 141,1\% en las privadas (IESALC, 2014). Nota Peña (2004) que el 50\% del total de matrículas se refería a las dos universidades más tradicionales.

A partir de 2005, año en que había 4 universidades públicas y 23 privadas, se verifica una inversión pues, según Alcaraz (2013), el 54\% de los estudiantes estaban matriculados en las universidades privadas y el $46 \%$ en las públicas. En 2008, las matrículas en el sector privado llegaron a 69\%, acompańando el crecimiento de las universidades privadas, que eran 35, en contraste con las 8 públicas. Mientras el número de universidades públicas no se alteró, la privatización se profundizó ya que en 2011, las matrículas alcanzaron el $81 \%$ y el número de instituciones privadas llegó a 44 (ALCARAZ, 2013). Observa Robledo (2016) importante aumento de inscriptos en las universidades privadas, entre los años 2011 y 2012, del $37,48 \%$ al 50,72\%, debido a factores como las vacantes escasas, los mecanismos de acceso más restrictos y la localización geográfica limitada de las universidades públicas. La autora también señala que el control de la calidad de las instituciones privadas era insuficiente, contribuyendo a la proliferación de ofertas de calidad dudosa.

El Paraguay compone el grupo de países en que el número de matrículas de la educación superior en el sector privado supera el 60\%, igual que en Brasil y Chile (LEMAITRE, 2014). Si durante 17 años, de 1990 hasta 2007, fueron creadas 29 universidades, 5 públicas y 24 privadas (RIVAROLA, 2008), en prácticamente un tercio de este tiempo, de 2007 a 2013, este número casi se ha duplicado, registrándose un total de 54 universidades, 8 públicas y 46 privadas, además de los 38 Institutos Superiores en todo el período (1990-2013), de los cuales 7 son públicos y 31 privados (ANEAES, 2014). 
En relación con la oferta de carreras, ésta se centraliza en ciertas áreas como derecho, administración, salud y formación de profesores (BRUNNER; HURTADO, 2011; ROBLEDO, 2016) en función de la empleabilidad, ya que los mayores empleadores son el Estado y el sector privado. El área tecnológica también se destaca por la demanda de carreras de informática debido al alta del sector de telecomunicaciones. Por otro lado, se observa el déficit de pertinencia en esta oferta, pues, no obstante Paraguay sea un país agroindustrial, no se verifica demanda en las carreras relacionadas directamente con estas actividades, así como ingeniería hidroeléctrica y la industria de construcción que necesitan mano de obra calificada (BRUNNER; HURTADO, 2011). Para Robledo (2016, p. 39): "los efectos de una desregulación excesiva, que se dio entre los años 2006 y 2009 ha dado origen a una oferta de baja calidad, hechos de estafa y corrupción que han alcanzado incluso a las dos universidades más tradicionales."

A partir de 2010, diversos actores sociales universitarios y no universitarios participaron de los foros de trabajo para identificar las cuestiones pendientes del sistema de educación superior, que se presentaba como "un conglomerado de instituciones, con escasa articulación entre ellas y menos aún, con la sociedad." (BRUNNER; HURTADO, 2011, p. 96) Como resultado de esta movilización social, fue sancionada la Ley 4.995 del 27 de mayo de 2013, la nueva Ley de Educación Superior, que trajo cambios sustanciales y definiciones imprescindibles para señalar los rumbos que la educación superior paraguaya pretende seguir, destacándose algunos a continuación.

En primer lugar, la ley declara la naturaleza de la educación superior, reconociéndola como un bien público, de responsabilidad del Estado y que constituye un derecho humano, garantizado a todos los que quieran y estén en condiciones legales y académicas de acceder. Esta premisa desencadena una serie de obligaciones del Estado en relación con la educación superior y su tratamiento legal, pues como bien público a ella no se aplican las reglas del mercado. La implementación de esta nueva ley de educación superior generó cambios significativos para el sector, especialmente con la creación del Consejo Nacional de Educación Superior (Cones), con atribuciones en el gobierno del sistema universitario, pudiendo proponer e implementar políticas públicas. Fue instalado en 2015 y tuvo diversos de- 
safíos, algunos de los cuales ocasionados por las movilizaciones estudiantiles generadas por graves irregularidades en las instituciones de educación superior, incluyendo las dos más prestigiosas del país, y culminando en una movilización nacional, que provocó cambios en casi todas las universidades públicas del país. En 2015, el Cones convocó al Congreso de Educación Superior 2015 que determinó la necesidad de asegurar la calidad de la Educación Superior en el Paraguay. (ROBLEDO, 2016)

\section{La evaluación y acreditación de la educación superior en Paraguay}

Con el término de la dictadura de Stroessner y, para adecuar la educación superior al nuevo contexto político y social, una serie de leyes fue promulgada desde la apertura del país, y en 1993, la más importante de esta etapa: la Ley de Universidades (Ley $n^{\circ}$ 136). Con esta Ley, fue instituido el principal órgano de regulación de las instituciones de enseńanza superior: el Consejo de Universidades, concentrando actividades relacionadas a la pertinencia de proyectos de creación de instituciones, a la formulación de la política de Educación Superior y a la coordinación y evaluación de la actividad universitaria en ámbito nacional, entre otras funciones. (PEÑA, 2004)

De esta forma, se delineó una primera regulación de la educación superior, adoptándose medidas como condicionar la autorización del Congreso Nacional para el funcionamiento de universidades a la existencia de un previo dictamen favorable y fundado del Consejo de Universidades y la exigencia de acreditación de requisitos mínimos para funcionamiento, expedida por el Consejo. Para dar más fuerza a la Ley de Universidades, las instituciones que no se adecuasen a sus preceptos no podían utilizar la denominación de Universidad o Facultad u otorgar diplomas, respondiendo ante la ley los responsables o directores de las instituciones que no cumplieran con la Ley. Sin embargo, el Consejo de Universidades no llegó a implantar este sistema de evaluación y acreditación, de acuerdo con Peña. (2004)

Así, esta iniciativa de los años noventa intentó introducir la incipiente cultura de la evaluación y acreditación en Paraguay y sufrió re- 
sistencias de los sectores más conservadores de las universidades. Pero, en 1998, se firmó el acuerdo regional de acreditación en el Mercosur para certificar la equivalencia de carreras de grado entre los países miembros y los Estados Asociados, con el objetivo de crear un sistema de acreditación futuro que posibilitase la circulación de recursos humanos calificados en el bloque. El Paraguay participó de esas discusiones sobre la acreditación regional en el Mercosur desde su inicio, en 1992, y, a pesar de la primera tentativa de establecer un sistema de evaluación con la ley de 1993 no haber tenido éxito, en 1998, la Ley General de Educación del país (Ley n ${ }^{\circ} 1264$ ) previó un proceso de evaluación sistemático para garantizar la calidad de la Educación, con responsabilidad de diferentes áreas: Ministerio de Educación y Cultura, gobernaciones, municipios y comunidades educativas.

Entre 1999 y 2001, fueron realizados talleres para abordar las temáticas de la evaluación y de la acreditación, de la formación de evaluadores y del sistema de créditos europeo, con la participación de expertos del Comité Nacional de Evaluación de Francia, de representantes de las instituciones ligadas al Mecanismo Experimental de Acreditación del Mercosur y de las Facultades de Agronomía, Ingeniería y Medicina de las universidades públicas y privadas (PEÑA, 2004). En el año 2000, el Ministerio de Educación y Cultura estableció la Comisión Técnica ad hoc y ad honorem para elaborar una propuesta de un modelo de Agencia de Evaluación y Acreditación, integrada por técnicos de la Dirección de Universidades e Instituciones del Ministerio y representantes de las universidades públicas y privadas (ANEAES, s/d).

De esta forma, las preocupaciones domésticas respecto de la calidad de la educación superior para la creación de un sistema nacional de evaluación y de acreditación de la educación superior se potencializaron con la participación del país en el proceso de acreditación regional del Mercosul (BARREYRO; LAGORIA; HIZUME, 2015). En consecuencia, en 2003, fue creada la Agencia Nacional de Evaluación y Acreditación de la Educación Superior (Aneaes), por la Ley no 2072/03. La Aneaes fue estructurada y configurada de acuerdo con los requisitos exigidos regionalmente para las Agencias Nacionales de Acreditación (ANAs), disfrutando de autonomía técnica y académica, aún cuando dependa del Ministerio de Educación y Cultura originalmente. La Agencia se autodefine como "ór- 
gano certificador de la calidad de la educación superior, en todos sus componentes." (ANEAES, s/d). Sus funciones son: a) realizar las evaluaciones externas de la calidad académica de las instituciones, b) producir informes sobre los proyectos académicos para la creación de cursos e instituciones, c) participar como órgano consultivo sobre evaluación y acreditación de la educación superior, d) asesorar a organismos e instituciones sobre la temática; e) acreditar la calidad académica de las carreras y programas de postgrado que hayan pasado por proceso de evaluación externa realizada por ella d) dar difusión pública acerca de las carreras acreditadas y e) vincularse con organismos nacionales o extranjeros para cooperar técnica e financieramente. (PARAGUAY, 2003, art. $4^{\circ}$ )

La Aneaes es regida por el Consejo Directivo, órgano colegiado compuesto por un miembro titular y uno suplente, nombrados por el Ministerio de Educación y Cultura; dos miembros titulares y dos suplentes, por el organismo que nuclea las instituciones de educación superior públicas y privadas; un miembro titular y uno suplente, nombrados por las federaciones de organizaciones de profesionales universitarios y un miembro titular y otro suplente, nombrados por las federaciones que conforman las asociaciones del sector productivo. Los cinco miembros son nombrados por Decreto del Poder Ejecutivo para ejercer esta función durante cuatro años (PARAGUAY, 2003). Interesante notar que en el primer período de funcionamiento del Consejo Directivo de la Agencia, el artículo 29 de la ley determinó que miembros de la Universidad Nacional de Asunción (UNA) y de la Universidad Católica Nuestra Señora de Asunción (UCA), las dos más tradicionales como ya se dijo, ocuparan los lugares destinados a las instituciones de educación públicas y privadas. El Consejo Directivo de la Agencia es el responsable de constituir el Registro Nacional de Pares Evaluadores por áreas disciplinarias o profesionales y de seleccionar y nombrar a los miembros de los Comités de Pares Evaluadores. Los pares extranjeros son elegidos por referencias de las distintas agencias que participan del proceso regional. Aún durante el período en que el Paraguay estuvo suspendido en el Mercosurb los pares de los otros Estados miembros continuaron participando del proceso paraguayo. (BARREYRO, 2014)

El modelo de evaluación de instituciones fue validado en el segundo semestre de 2009 (ANEAES, s/d). Ya el Modelo Nacional de Acreditación para carreras de grado fue diseñado por la Aneaes, con la asistencia de 
expertos del Centro Interuniversitario de Desarrollo Andino, (Cinda), de Chile, en 2007 (ROBLEDO, 2016). La acreditación es definida como "la certificación de calidad académica", "basada en un juicio sobre la consistencia entre los objetivos, los recursos y la gestión de una unidad académica" (PARAGUAY, 2003, art. 22 $2^{\circ}$ realizada en las carreras y programas de educación que ya posean egresos, comprendiendo tres etapas: la autoevaluación, la evaluación externa y el informe final, como en el proceso regional. La participación en los procesos de evaluación externa y acreditación era obligatoria para las carreras de arquitectura, derecho, ingeniería, ingeniería agronómica, medicina, odontología, y todas aquellas cuyos títulos habiliten para el ejercicio profesional de prácticas que puedan ocasionar daños a la integridad de las personas o a su patrimonio. También se implantó, la acreditación de instituciones de educación superior y de cursos de postgrado, con carácter voluntario. (ROBLEDO, 2016)

Pero este sistema no cubría todo el sistema pues sólo fue aplicado en las carreras para las cuales la acreditación era obligatoria. El descontrol en la abertura de instituciones derivó en un gran número de carreras de dudosa calidad y generó demanda al Estado para asumir su papel de agente regulador de la educación superior (BRUNNER; HURTADO, 2011; ROBLEDO, 2016). Con la nueva Ley de Educación Superior, en 2013, se preveía la obligatoriedad de la evaluación y de la acreditación, la posibilidad de intervención y de cierre de universidades, institutos superiores e institutos de formación profesional del tercer nivel, la limitación taxativa de la autonomía universitaria, el aumento de poderes y autonomía financiera de la Aneaes y la reglamentación de las actividades docentes.

La Aneaes ganó más importancia con la Ley de Educación Superior, pues fue transformada en órgano efectivo de control del sistema de educación superior y de apoyo en la regulación, debido a que la acreditación se convirtió en un requisito de acceso a: a) fondos públicos y becas del Estado, b) participación en concursos, licitaciones y c) prestación de servicios públicos (ROBLEDO, 2016). Las Instituciones de Educación Superior que estaban en funcionamiento, al entrar en vigencia la nueva Ley, tuvieron dos años de plazo para adecuarse a sus exigencias (PARAGUAY, 2013).

Los procesos de acreditación en Paraguay presentan diversas peculiaridades. Por ejemplo, mientras el sistema regional tiene cuatro dimensiones, el paraguayo tiene cinco. La quinta se refiere a los resultados y el 
impacto, y la carrera evalúa sus egresados, mediante un plan de seguimiento de egresados, la retroalimentación de su plan curricular y un proyecto educativo basado en lo que los egresados y los empleadores sugieren a las carreras. Otra cuestión es que en el sistema paraguayo se da más énfasis a la docencia en la formación profesional y en el Mercosur, a la investigación (BARREYRO, 2014). Sin embargo, la principal característica del sistema paraguayo era que aplicaba los procesos de evaluación nacional simultáneamente a los del Mercosur, pues el comité de pares se conforma con dos pares internacionales y un par nacional. La idea es que la participación de pares extranjeros trae más objetividad y credibilidad al proceso nacional ya que la mayoría de los docentes de una carrera se conocen porque el sistema es de tamaño medio-pequeño. Como no todas las carreras son acreditadas regionalmente, en los casos en que la carrera está inscrita en ambos procesos, los pares evalúan también la dimensión egresos. Además, los requerimientos para participar en los dos sistemas no son los mismos: por ejemplo, en el modelo regional se requieren cinco promociones de egresados y en el nacional, basta una sola. Así, pocas carreras pasan por los dos procesos de acreditación. La Aneaes paga a los evaluadores los pasajes, los viáticos y el peaje, con los fondos recaudados del arancel que las carreras pagan para acreditarse, cerca de U\$S 7.000.00 (BARREYRO, 2014).

Sin embargo, hubo importantes cambios en 2016, pues la Resolución $n^{\circ} 312$ de la Aneaes alteró el procedimiento estableciendo la necesidad de contar con la acreditación vigente en el proceso nacional, como requisito para la inscripción en el proceso de acreditación regional. Así, con la publicación de las primeras convocatorias con estas nuevas características, en 2017, Paraguay pasa a tener dos procesos de acreditación distintos: el nacional y el regional (ANEAES, 2016). Hasta el momento, Paraguay tiene 114 carreras de grado y 5 de postgrado (todas en pediatría médica) acreditadas por el sistema nacional (ANEAES, s/d, b, c).

Otra consecuencia de esa ley fue el Acta $n^{\circ} 1$ del 18 de enero de 2016, del Consejo Directivo de la Aneaes, que exime a las universidades públicas de pagar aranceles para la acreditación, como reconocimiento de la educación superior como un bien público y como forma de garantizar su calidad, tanto en el sistema nacional como en el regional. (ANEAES, 2016a) 


\section{La participación paraguaya en la acreditación regional de carreras}

Como miembro fundador del Mercosur, instituido por el Tratado de Asunción de 1991, Paraguay participó desde el inicio de las actividades del Sector Educativo y de las proposiciones para la creación de un sistema regional de acreditación de carreras iniciadas en 1992, aun cuando su sistema de educación superior estaba en pleno desarrollo y sufriendo profundas transformaciones. Como en aquel momento en el nivel nacional no existía la práctica de la evaluación y la acreditación de carreras, fue nombrada una Comisión para representar al país en el Grupo de Trabajo de Especialistas en Evaluación y Acreditación del bloque, formada por integrantes del Ministerio de Educación y Cultura y del Consejo de Universidades (PEÑA, 2004).

Una vez firmado el Memorando de Entendimiento sobre la Implementación de un Mecanismo Experimental de Acreditación de Carreras para el Reconocimiento de Títulos de Grado Universitario en los Estados Partes del Mercosur, Bolivia y Chile (Mexa) en 1998, Paraguay acordó que debería ajustarse a los movimientos regionales y adoptar un modelo similar a los de Argentina, Brasil y Chile, empezando, por ende, los trámites internos para la creación de un sistema nacional de acreditación de carreras, según Robledo y Caillón. (2009)

Mientras en el nivel nacional los trabajos sobre evaluación y acreditación se iniciaban, Paraguay participaba del pre-test, aplicado en 2000, para verificar la adecuación de los criterios e indicadores establecidos en el Mexa. Cada país nombró dos instituciones para participar del proceso de acreditación experimental de las carreras de agronomía, ingeniería y medicina y las dos universidades más tradicionales fueron las escogidas. La Universidad Nacional de Asunción tuvo las carreras de agronomía y medicina acreditadas y la Universidad Católica, la de ingeniería (HERMO, 2011). Señala Real (2013) que la Aneaes tuvo el apoyo del Banco Interamericano de Desarrollo (BID) para la implementación de los primeros procesos de evaluación regionales, así como de la Comisión Nacional de Acreditación (CNAP) del Ministerio de Educación de Chile.

Para participar del Mecanismo Experimental de Acreditación de Carreras de Grado Universitario (Mexa) del Mercosur, el Poder Ejecutivo 
creó una Comisión ad hoc para desarrollar el proceso regional en el país, asumiendo así las funciones de Agencia Nacional de Acreditación (ANA). Esta Comisión inició sus trabajos en 2002, con el lanzamiento de la convocatoria para las carreras de Agronomía (PEÑA, 2004) y actuó en el Mexa hasta que la Agencia Nacional de Acreditación y Evaluación de la Educación Superior (Aneaes) pudo funcionar plenamente, lo que ocurrió en noviembre de 2003. (ANEAES, s/d)

Así, la Aneaes comenzó a funcionar ejecutando acciones del Mexa, desde finales de 2003. En la aplicación del Mecanismo, que concluyó en 2006, el Paraguay tuvo 7 carreras acreditadas (Cuadro 1).

\begin{tabular}{|c|c|c|}
\hline Carrera & \multicolumn{2}{|c|}{ Universidad } \\
\hline Agronomía & \multicolumn{2}{|c|}{ UNA } \\
\hline \multirow{3}{*}{ Ingeniería } & \multirow{2}{*}{ UNA } & Civil \\
\cline { 2 - 3 } & & Electrónica \\
\cline { 2 - 3 } & \multirow{2}{*}{ UC } & Electromecánica \\
\cline { 2 - 3 } & & Civil \\
\hline Medicina & & ENA \\
\hline
\end{tabular}

Cuadro 1: Carreras Acreditadas en el Mexa

Fuente: Mercosul (2004) y Mercosur (2006) Elaboración de las autoras.

Con base en el Mexa, en 2008 fue firmado el Acuerdo sobre la Creación e Implementación de un Sistema de Acreditación de Carreras Universitarias para el Reconocimiento Regional de la Calidad Académica de las Respectivas Titulaciones en el Mercosur y Estados Asociados (Sistema Arcu-Sur). Además de las carreras de agronomía, ingeniería y medicina, fueron agregadas otras cuatro carreras: arquitectura, enfermería, odontología y veterinaria.

La Aneaes empezó los trabajos del primer ciclo de acreditaciones del Sistema Arcu-Sur en 2008, ajustando las convocatorias nacionales a las regionales, realizando llamadas conjuntas para el Modelo Nacional y el Sistema Arcu-Sur en todas las carreras (ANEAES, s/d). De las carreras acreditadas por el Mexa, solamente la de ingeniería civil de la Universidad 
Católica no fue re-acreditada, expirando su acreditación el 2 de junio de 2011. (SISTEMA ARCU-SUL, s/d)

Hasta 2012, cuando Paraguay fue suspendido del Mercosur y debió retirarse de todas las actividades, inclusive del Sistema Arcu-Sur, 17 carreras habían sido acreditadas (Tabla 1).

Tabla 1: Carreras acreditadas por el Sistema Arcu-Sur

\begin{tabular}{|c|c|c|c|}
\hline Carrera & \multicolumn{2}{|c|}{ Universidad } & Total \\
\hline Agronomía & \multicolumn{2}{|c|}{$\begin{array}{l}\text { Universidad Nacional de Asunción } \\
\text { (UNA) }\end{array}$} & 1 \\
\hline \multirow[b]{2}{*}{ Arquitectura } & \multicolumn{2}{|c|}{ UNA } & \multirow[b]{2}{*}{2} \\
\hline & \multicolumn{2}{|c|}{$\begin{array}{c}\text { Universidad Católica Nuestra Señora de } \\
\text { Asunción (UCA) }\end{array}$} & \\
\hline \multirow{2}{*}{ Enfermería } & \multicolumn{2}{|c|}{ UNA } & \multirow{2}{*}{2} \\
\hline & \multicolumn{2}{|c|}{ UCA } & \\
\hline \multirow{7}{*}{ Ingeniería } & \multirow{5}{*}{ UNA } & Agronómica & \multirow{7}{*}{7} \\
\hline & & Civil & \\
\hline & & Electromecánica & \\
\hline & & Industrial & \\
\hline & & Química & \\
\hline & \multirow{2}{*}{ UCA } & Electrónica & \\
\hline & & Industrial & \\
\hline \multirow{3}{*}{ Medicina } & \multicolumn{2}{|c|}{ UNA (Guairá) } & \multirow{3}{*}{3} \\
\hline & & $\mathrm{CA}$ & \\
\hline & \multicolumn{2}{|c|}{ Universidad Nacional de Itapúa } & \\
\hline Odontología & \multicolumn{2}{|c|}{ UNA } & 1 \\
\hline Veterinária & \multirow{2}{*}{\multicolumn{2}{|c|}{ UNA }} & 1 \\
\hline Total & & & 17 \\
\hline
\end{tabular}

Fuente: Sistema Arcu-Sul (2017). Elaboración de las autoras.

La última acreditación realizada por el país, en el primer ciclo del Sistema Arcu-Sur, fue la de la carrera de Odontología, el 15 de noviembre de 2011. Durante la suspensión del país en el Mercosur, las convo- 
catorias para acreditación pasaran a contemplar solamente el proceso nacional (BARREYRO, 2014). La reintegración de Paraguay al proceso regional de acreditación se dio en la reunión de la Red de las Agencias Nacionales de Acreditación (Rana), realizada entre 18 y 19 de agosto de 2014 (MERCOSUR, 2014).

Actualmente, Paraguay tiene 10 carreras acreditadas y 8 con las acreditaciones caducadas por el Sistema Arcu-Sur (SISTEMA ARCU-SUL, s/d) y no ha lanzado convocatorias para el segundo ciclo que debería incluir tres carreras más: farmacia, geología y economía. (MERCOSUR, 2014)

\section{Consideraciones finales}

La evaluación de la educación superior en el Paraguay ha estado en la agenda desde 1998 sufriendo los avatares de un país que pasó por numerosos episodios políticos desde entonces y recibiendo la influencia de procesos políticos regionales de integración.

Así, la acreditación de la educación superior en el Paraguay muestra la influencia de los procesos de acreditación desarrollados en la escala regional del Mercosur, de los que el país participó desde su inicio, como catalizadores del desarrollo de los procesos nacionales. Puede detectarse que la escala nacional de la evaluación se desarrolló simultáneamente con la escala regional, dado que el país creó una agencia de evaluación siguiendo el modelo de las Agencias Nacionales de Acreditación del MERCOSUR y fue ella la que implementó los procesos regionales, a los que se sumaron los nacionales. Los dos procesos se desarrollaban en el mismo momento, siendo que en la escala nacional apenas se incluyó un ítem más a los propuestos por la escala regional.

Para Robledo y Caillón (2009), lo que inicialmente podría ser configurado como un retraso de Paraguay en relación a los sistemas educativos de los países vecinos, como Argentina y Brasil, se transformó en una ventaja, pues, su sistema de acreditación nacional buscó la mayor convergencia posible con el proceso de acreditación regional. En consecuencia, según las autoras, los standards de evaluación y acreditación de las universidades paraguayas corresponden, mínimamente, a las expectativas de garantía de 
calidad indicadas por los expertos del bloque y de los Estados Asociados, confiriéndole, incluso, mayor credibilidad.

En 2016, se registró un importante cambio, con el requisito de previa acreditación en los procesos nacionales para participar de los regionales. Esto puede entenderse como una apropiación de la acreditación en la escala nacional, después de los cambios en la educación generados por reclamos de mejoría de calidad de la educación. Pero no deja de ser paradójico que justamente ocurra cuando el segundo ciclo del sistema Arcu-Sul se inició con la intencionalidad de que los procesos en la escala nacional y la regional no fuesen duplicados, lo que podría parecer una involución en el caso del Paraguay si no se tuviera en cuenta el contexto en que surgió, que es el de la preocupación de la sociedad por la calidad de su educación superior, no obstante, el proceso regional haya quedado en segundo plano.

\section{Notas}

1 El texto es producto de la investigación Acreditacão da educação superior no Mercosul: o papel do Brasil, que fue apoyada por el CNPQ (Edital Universal Proceso $n^{\circ}$. 483.258/2011-3) y se basó en documentos y entrevistas que siguieron los procedimientos éticos establecidos para la investigación científica en Ciencias Humanas.

2 El país tiene una población de 6.993.000 habitantes (CEPAL, s/d). y es uno de los más desiguales del mundo, presentando un índice de Gini mayor o igual a 0.50 (BRUNNER; HURTADO, 2011). Su tasa de alfabetización es del $99 \%$, y su gasto con educación es del $5 \%$ del PBI (CEPAL, s/d). El sistema educativo paraguayo está compuesto por: educación inicial, escolar básica, permanente, media, técnica, indígena y superior La educación escolar básica se imparte entre los 6 y 14 años de edad y es obligatoria y gratuita en las escuelas públicas de gestión oficial. (PARAGUAY, 2014b)

3 De 1954 a 1989, el Paraguay estuvo bajo la dictadura militar del General Alfredo Stroessner. Este gobierno fue el segundo más largo de la historia de América Latina en el siglo XX, después del gobierno de Fidel Castro en Cuba.

4 Brunner y Hurtado (2011) consideran los tamaños de los sistemas de educación superior, a partir de las matrículas, de la siguiente forma: pequeño ( 0 a 150 mil); medio-pequeño (150 a 500 mil); medio (500 a 1 millón); medio-grande (1 a 2 millones) y grande (más de 2 millones).

5 En este apartado, se presentan algunos de los pocos datos cuantitativos sobre la educación superior paraguaya, disponibles en fuentes primarias y secundarias, dado que "la falta de un sistema de información fiable y actualizada es una deuda pendiente de todo el sistema educativo." (ROBLEDO, 2016, p.14)

6 Entre junio de 2012 y agosto de 2013 el país fue suspendido del Mercosur, debido al juicio político realizado al entonces presidente Fernando Lugo, calificado de ilegítimo por no haberse respetado el debido proceso. Después de nuevas elecciones democráticas, el Paraguay fue reincorporado. 


\section{Referencias}

ALCARAZ, José Nicolás Morínigo. La educación universitaria en el sistema educativo paraguayo. In: Pensar la universidad. La educación superior universitaria y su marco regulatorio. Programa de Democracia Sociedad Civil Topu'á Paraguay. Asunción, 2013, p. 24-31. Disponible en: < http://topua.semillas.org.py/wp-ontent/uploads/2011/01/ Pensarlauniversidad.pdf >. Acceso en: 10 mar. 2014.

ANEAES. Instituciones de educación superior, Paraguay, 2014. Disponible en: <http:// www.aneaes.gov.py/aneaes/index.php/ct-menu-item-31>. Acceso en: 5 mar 2014.

ANEAES. La creación de la Agencia Nacional de Evaluación y Acreditación de la Educación Superior (ANEAES) (s/d). Disponible en: <http://www.aneaes.gov.py/aneaes/ index.php/ct-menu-item-2/ct-menu-item-3>. Acceso en: 10 abril 2015.

ANEAES. (s/d, a) Resolución no 212 de 18 de julio de 2016. Disponible en: <http:// www.aneaes.gov.py/aneaes/datos/modelo_nacional/resolucion212.pdf >. Acceso en: 5 de mar. 2017.

ANEAES (s/d, b). Carreras acreditadas de grado. Disponible em: <http://www.aneaes. gov.py/aneaes/index.php/ct-menu-item-12/carreras-acreditadas> . Acceso en: 15 mar. 2017.

ANEAES (s/d, c). Carreras de posgrado acreditadas. Disponible en: <http://www.aneaes. gov.py/aneaes/index.php/programas-de-posgrado-acreditados $>$. Acceso en: 15 mar. 2017.

BARREYRO, Gladys Beatriz. Acreditação da Educação Superior no Mercosul:o papel do Brasil. Relatório final de pesquisa. Edital Universal CNPQ/Processo n. 483.258/2011-3 (mimeo), 2014.

BARREYRO, Gladys Beatriz, LAGORIA, Silvana Lorena; HIZUME, Gabriella de Camargo. As agências nacionais de acreditação no Sistema ARCU-SUL: primeiras considerações. Avaliação, Campinas-Sorocaba. v. 20, n. 1, p. 49-72, 2015.

BRUNER, José Joaquín; HURTADO, R. F. (Coord.). Educación Superior en Iberoamerica. Informe 2011. Centro Interuniversitario de Desarrollo Andino (CINDA). Disponible en: <http://www.iesalc.unesco.org.ve/index.php?option=com_content\&view =article\&id=2460\&Itemid=1389\&lang=es $>$. Acceso en: 10 mar. 2015.

COMISIÓN ECONÓMICA PARA AMÉRICA LATINA Y CARIBE (CEPAL). Paraguay: perfil nacional socio-demográfico (s/d, . Disponible en: http://interwp.cepal. org/cepalstat/WEB_cepalstat/Perfil_nacional_social.asp?Pais=PRY\&idioma=e>. Acceso en: 7 abril 2015.

HERMO, Javier Pablo. La acreditación regional de carreras en el Mercosur: presente e futuro. Saarbrücken: Editorial Académica Española, 2011. 


\section{INSTITUTO INTERNACIONAL PARA LA EDUCACIÓN SUPERIOR EN} AMÉRICA LATINA Y CARIBE (IESALC). La educación superior pública y privada en América Latina y Caribe. Contexto de internacionalización y proyecciones de políticas públicas. 2014. Disponible en: <http://www.iesalc.unesco.org.ve/index. php?option=com_content $\&$ view $=$ article $\&$ id $=2460 \&$ Itemid $=1389 \&$ lang=es $>$. Acceso en: 10 abril 2015.

LEMAITRE, María José. Mapa de temas críticos en el debate de la Educación Superior Pública y Educación Privada en América Latina y el Caribe. In: UNESCO-IESALC. La Educación Superior Pública y Privada en América Latina y Caribe. Contexto de internacionalización y proyecciones de Políticas Públicas, 2014, p. 132-154. Disponible en:<http://www.iesalc.unesco.org.ve/index.php?option=com_fabrik\&view=details\&for mid=2\&rowid=173\&lang=es $>$. Acceso en: 10 abril 2015.

MERCOSUL. Conselho Mercado Comum. Reunião dos Ministros de Educação. Acta 02/2004.

MERCOSUR. Reunión de Ministros de Educación. Acta 01/2006.

PARAGUAY. Ley n. 4995 de 27 de junio de 2013. Ley de la Educación Superior. Disponible en: <http://www.sansebastian.edu.py/uassweb/file/Ley_4995_De_ Educacion_Superior.pdf. Acceso en: 10 abril 2015.

PARAGUAY. Ley n. 2072 de 13 de febrero de 2003. Ley de Creación de la Agencia Nacional de Evaluación y Acreditación de la Educación Superior. Disponible en: < http://www.pol.una.py/sites/default/files/files/reglamentos/Ley2072DeLaANEAES. pdf>. Acceso en: 10 de abril 2015.

PARAGUAY. Ministerio de Educación y Cultura. Educación Superior (2014a). Disponible en: < http://www.mec.gov.py/cms >. Acesso en: 7 abril 2015.

PARAGUAY. Ministerio de Educación y Cultura. El sistema educativo (2014b). Disponible en:<http://www.mec.gov.py/cms>. Acceso en: 7 abril 2015.

PEÑA, Haydée Gimenez. El proceso de acreditación de carreras en Paraguay. In: La Evaluación y Acreditación Superior en América Latina y Caribe. Venezuela: IESALC, 2004, p. 191-207. Disponible en: <://www.iesalc.unesco.org.ve/index.php?option=com_ fabrik\&view=details\&formid=2\&rowid=46\&lang=es $>$. Acceso en: 2 nov. 2009.

RIVAROLA, Domingo. La universidad paraguaya, hoy. Avaliação (Campinas), v. 13-2. p. 533-578, jul. 2008. Disponible en: <http://www.scielo.br/scielo.php?script=sci_ pdf\&pid $=$ S1414-40772008000200014\&lng=en\&nrm=iso\&tlng=es $>$. Acceso en: 10 abril 2015.

REAL, Gisele Cristina Martins. Avaliação da educação superior no Brasil e no Paraguai: implicaçóes para a construção do Mercosul Educacional. Tópicos Educacionais, Recife, n.1, jan-jun, 2013, p. 7-25. 
ROBLEDO, Rocío. Educación Superior en Iberoamérica: informe 2016. Informe nacional: Paraguay. Chile: Cinda, 2016. Disponible en: < http://www.cinda. cl/2016/11/16/informe-educacion-superior-en-iberoamerica-2016/\#more>. Acceso en: 7 fev. 2017.

ROBLEDO, Rocío; CAILLÓN, Adriana; Procesos regionales en educación superior. El mecanismo de acreditación de carreras universitarias en el MERCOSUL.

Reconocimiento regional de los títulos y de la calidad de la formación. In: Educación Superior y Sociedad. Experiencias de convergencia académica en los países del Mercosur. Nueva Época. IESALC. Año 14, n. 1, p.73-98.

SISTEMA ARCU-SUL (s/d). Acreditação regional de cursos universitários Mercosul. Disponible en: <http://sistemaarcusul.mec.gov.br/arcusul/pages/pesquisaexterna/ pesquisarCursoExterno.seam;jsessionid=8ysfpcm5TVHqW2fyq31uFw_.

node01\#tabelaCursos>. Acceso en: 19 mar. 2017.

Recebido em 22 mai. 2018 / Aprovado em I2 nov. 2018

\section{Para referenciar este texto:}

BARREYRO, G. B.; HIZUME, G. C. el Paraguay y la acreditación de carreras de grado en el Mercosur. EccoS - Revista Cientifica, São Paulo, n. 47, p. 41-59. set/dez. 2018. Disponível em: <https://doi.org/10.5585/EccoS.n47.8701>. 
